\title{
A New Variable Stiffness Design: Matching Requirements of the Next Robot Generation
}

\author{
Sebastian Wolf and Gerd Hirzinger \\ DLR - German Aerospace Center \\ Institute of Robotics and Mechatronics \\ D-82234 Wessling, Germany \\ \{sebastian.wolf, gerd.hirzinger\}edlr.de
}

\begin{abstract}
Facing new tasks, the conventional rigid design of robotic joints has come to its limits. Operating in unknown environments current robots are prone to failure when hitting unforeseen rigid obstacles. Moreover, safety constraints are a major aspect for robots interacting with humans. In order to operate safely, existing robotic systems in this field are slow and have a lack of performance. To circumvent these limitations, a new robot joint with a variable stiffness approach (VS-Joint) is presented. It combines a compact and highly integrated design with high performance actuation. The VSJoint features a highly dynamic stiffness adjustment along with a mechanically programmable system behavior. This allows an easy adaption to a big variety of tasks. A benefit of the joint is its intrinsic robustness against impacts and hard contacts, which permits faster trajectories and handling. Thus, it provides excellent attributes for the use in shoulder and elbow joints of an anthropomorphic robot arm.
\end{abstract}

\section{INTRODUCTION}

In the current robotics research a main field is focused on the development of joints with variable mechanical impedance, see Fig. 1, [1]-[10]. The ambition of this development is to bring the robots closer to the human and even in direct contact for hand to hand interaction. The robots are to leave their separated space and assist the humans, maybe even at their home. Therefore, the robots have to be more gentle to their environment, but also have to be strong enough to fulfill their purpose. Since humans designed their surroundings for their own ergonomics, robots have to have similar abilities for a skilled and useful assistance.

There are several reasons for building a robot with mechanically compliant joints like in [2]. Up to now the main reason to deliberately introduce joint compliance was to ensure safety to the human user. However, in [11] it is stated that decreasing joint stiffness is an adequate countermeasure to reduce joint torques during rigid and fast impacts with hard surfaces and therefore protecting the robot as well. The bandwidth of the compliance, which can be achieved by control with rigid joints, is limited because of the time delay in the sensor data acquisition, the control loop, and the motor inertia [8]. Hard impacts result in high contact forces and torques at a very small position difference between motor

This work has been partially funded by the European Commission's Sixth Framework Programme as part of the projects SMERobot ${ }^{\text {TM }}$ under grant no. 011838 and PHRIENDS under grant no. 045359.

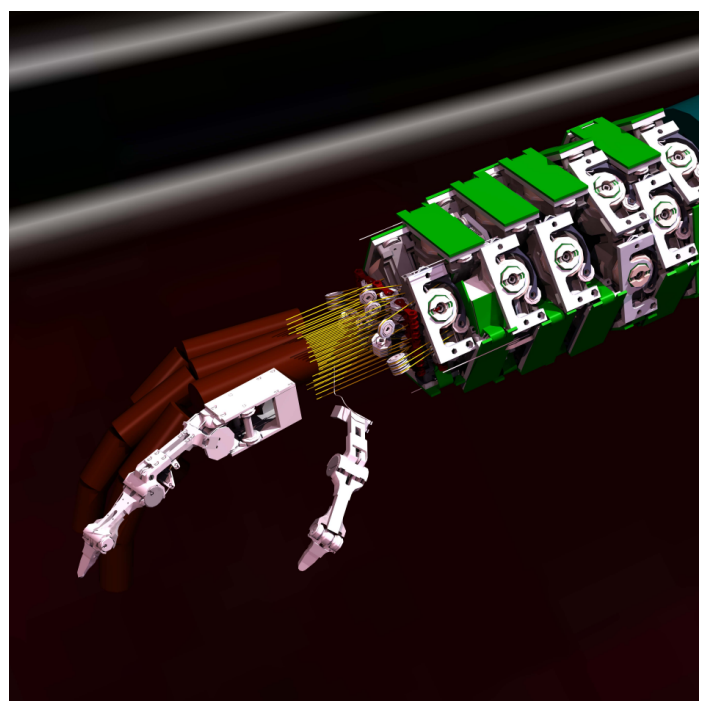

Fig. 1. Design study of DLR's integrated Hand-Arm-System

and link. The reaction time of the robot limits the speed of the trajectory to ensure that the force and torque limits are not exceeded. Lower stiffness in the joint provides a longer time span to react to the impact and to avoid an external overload. The balancing of the external torque by the mechanical compliance allows to move on faster trajectories without exceeding the safety limits but has a loss in the system accuracy.

Besides of the safety aspect, a flexible joint has an advantage in the system performance compared to a stiff joint. The flexibility itself can be seen as a mechanical energy storage, or capacitor. It can be used for buffering external or motor torque, and if the additional inertia is kept small, the bandwidth of the compliance is almost the same as the one of the decoupled system. With adequately planed trajectories the stored energy can be added where required to the mechanical energy supplied by the joint motor. This enables the link to be accelerated to a much higher peak velocity than the maximum joint motor speed and thus enhances the joint performance significantly.

Introducing a possibility to change the stiffness of the joint while the robot is operating permits a further enhancement 
of the skills of a joint [3]. Similar to a human, who can change the stiffness of his joints by straining the agonist and the antagonist, the stiffness can be changed according to the performed task. One approach for a variable stiffness is an antagonistic system like in the natural archetype, which is successfully implemented in [4], [5], [6]. Two opposing actuators of similar size and series elastic elements drive one link by moving in the same direction and change the joint stiffness by moving in the opposite direction. In every case the friction of both motors and maybe the spring mechanisms determine an energy loss. Furthermore, unless less efficient non-backdrivable gears are used, a high stiffness setting demands a constant torque of both actuators in opposing directions. This has also some drawbacks in the energy consumption. The approach in [7] aims at a reduction of these effects by motor cross-coupling. However, an antagonistic system is capable to distribute the power of both motors completely to the change of stiffness. Using a setup in which the motors are not opposed in the antagonistical way promise to have less energy consumption, a smaller volume and lower mass [8], [9], [10]. This of course depends on the design and the desired task. When the mechanical behavior of the system can be adjusted close to the desired overall system behavior, it is possible to reduce the control effort with preexisting knowledge of the desired application (impedance matching). Especially for cyclic motions and trajectories, in which the link has to be stopped and accelerated in the opposite direction like walking, running, or throwing, a preset can be given to the system according to the applied load and speed [12], [13], [14]. In some cases one stiffness preset is enough for the whole performed application, but in a real environment the robot has to adapt its stiffness to changing objects and desired tasks. In this case a continuous and fast change of the stiffness setup is needed. Compared to a conventional robot like the DLR Justin [15], the stiffness of a variable stiffness joint is still orders of magnitude less. In an unknown environment with the possibility of sudden impacts, the joint will be set to a stiff setup to prevent the joint from overload and running into the hardware limits. High stiffness will provide better results in a precise positioning task. In contrary a soft preset will be the best choice for a gentle manipulation in a sensitive environment.

The previous considerations are leading to the development of the variable stiffness joint (VS-Joint) presented in the following sections. Compared to state-of-the-art systems the new development addresses particularly the performance, compactness, and friction of the system.

\section{VS-JOINT MECHANICS}

\section{A. Requirements}

The aim of the development of the new VS-Joint (patent pending) is to introduce a mechanical passive compliance into a robot joint. It should be possible to change the stiffness of the joint continuously and with the maximum load applied. The maximum output torque should be at least $120 \mathrm{Nm}$. Other design goals are low weight, and a compact and robust mechanics, which allows the assembly in a robot arm system

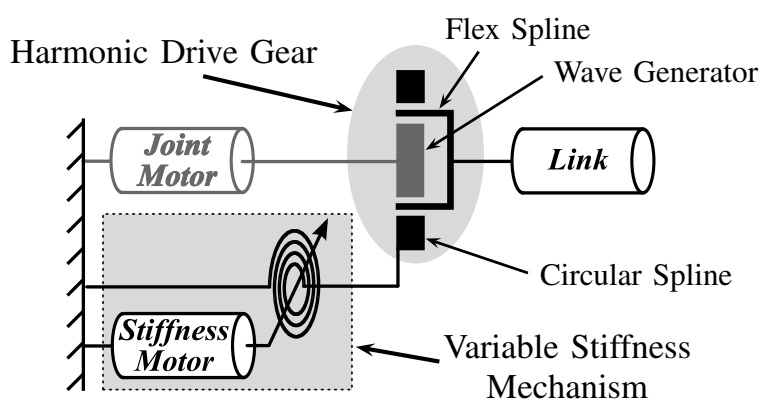

Fig. 2. Principle of variable stiffness joint mechanics. The circular spline of the harmonic drive gear is supported by the new mechanism.

of the size of a human arm. Low friction and inertia at the link side are required for a high bandwidth of the spring mechanism and a low energy loss in operation.

\section{B. Design}

The concept of the VS-Joint is based on two motors of different size to change the link position and the stiffness preset separately, see Fig. 2. The high power motor changes the link position and is connected to the link via a harmonic drive gear. Mechanical compliance is introduced by the VSJoint mechanism, which forms a flexible rotational support between the harmonic drive gear and the joint base. The joint stiffness is changed by a much smaller and lighter motor, which changes the characteristic of the supporting mechanism.

The harmonic drive gear consists of three main parts. In a standard setup the wave generator (WG) is connected to the motor axle, the flex spline (FS) is attached to the link and the circular spline (CS) is fixed to the base of the joint. In the VS-Joint the circular spline is pivoted. The mechanism of the VS-Joint acts as a spring like support between the circular spline and the joint base. In case of a passive compliant deflection $\varphi$ of the joint, the CS and the FS rotate relative to the base. The formula for the gear motion with a nominal transmission ratio of $100 / 1$ is given in (1), where the angle indices are the corresponding gear part names.

$$
\vartheta_{\mathrm{CS}}=\frac{100}{101} \vartheta_{\mathrm{FS}}+\frac{1}{101} \vartheta_{\mathrm{WG}}
$$

The VS-Joint mechanism provides a centering torque $\tau$ against the compliant joint deflection. The extent of the torque can be influenced by the stiffness actuator. The mechanism transforms the rotation of the CS into a linear motion of a slider, see Fig. 3. This is done by 4 cam rollers running on a rotationally symmetric cam disk, which is connected to the CS. The cam rollers are connected to the slider, which is guided by linear bearings in axial direction. A motion of the slider compresses 4 spiral springs, which results in a force on the cam rollers, see Fig. 4. The force is transmitted by the cam rollers to the cam disk and results in a centering torque. The force of the springs can be increased by moving the spring base towards the cam disk. The spring base is realized in the form of a second slider. Preload is created by moving the spring base slider via a spindle 


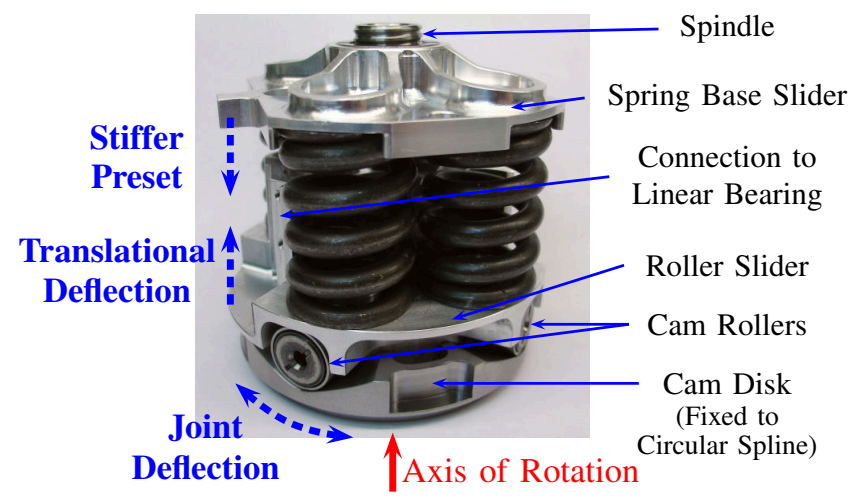

Fig. 3. VS-Joint mechanism. The joint axis is in the vertical direction. The cam disk rotates on a compliant joint deflection according to (1) which results in a vertical displacement of the roller slider. A stiffer joint preset is achieved by moving the spring base downward.

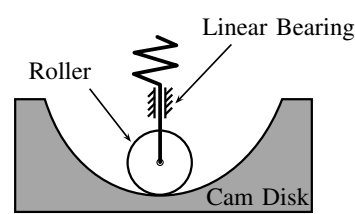

(a)

Fig. 4. Unwinded schematic of the VS-Joint principle in centered (a) and deflected (b) position. A deflection of the joint results in a horizontal movement of the cam disk and a vertical displacement of the roller. The spring force generates a centering torque on the cam disk

attached to the stiffness adjusting motor (Maxxon EC22 with an intermediary planetary gear).

Concerning passive spring deflection and active joint movement, the location of the VS-Mechanism has significant benefits regarding the system inertia and the resulting bandwidth. The main parts of the mechanism are rotationally fixed to the joint base. A passive deflection rotates only the $\mathrm{CS}$, the cam disk, and its bearing together with the link. The added inertia of these three parts is kept very low (see Table I) and the joint motor with the WG are not moved. The torque of an active joint movement is transferred directly via the gear from the joint motor to the link without additional friction and inertia of the VS-Mechanics.

The cam disk can have different kind of shapes. A concave shape results in a progressive, a convex in a degressive, and a linear in a linear system behavior. By shaping the cam disk in a concave way with a radius lower or the same as the

TABLE I

VS-JOINT PROPERTIES

\begin{tabular}{cc}
\hline Max. Torque & $160 \mathrm{Nm}$ \\
Max. Deflection & $\pm 14^{\circ}$ \\
Diameter & $97 \mathrm{~mm}$ \\
Length & $106 \mathrm{~mm}$ \\
Weight (incl. stiffness adjuster) & $1.4 \mathrm{~kg}$ \\
Link Side Inertia & $2.34 \times 10^{-4} \mathrm{~kg} \mathrm{~m}^{2}$ \\
\hline
\end{tabular}

cam rollers, the system torque behavior at this point will be a jump or a resting point respectively. It can be overcome by a torque rising above a certain threshold. The shape of the cam disk can also be designed to have a different system behavior depending on the deflection direction.

\section{Layout}

Several cam disks have been built, however, in the following only one cam disk with a symmetric concave shape of a constant radius $R=19 \mathrm{~mm}$ will be discussed. The cam rollers have a radius $r=8 \mathrm{~mm}$ and roll on a radius $c=33 \mathrm{~mm}$ relative to the joint axis. The springs have a overall spring constant of $k=908 \mathrm{~N} / \mathrm{mm}$. The stiffness adjusting motor position $\sigma$ is limited to $\sigma_{\max }=630^{\circ}$, which will be considered as $100 \%$ in the following.

In the unwinded model of the system the joint deflection is $c \varphi$ and the angle $\alpha$ is:

$$
\alpha=\sin ^{-1}\left(\frac{c \varphi}{R-r}\right)
$$

The displacement of the cam rollers $y$ in the direction of the joint axis

$$
y=(R-r)(1-\cos \alpha)
$$

and the displacement of the stiffness adjusting slider result in the compression of the springs. By multiplying this displacement with the spring constant the spring force results

$$
F=k\left((R-r)(1-\cos \alpha)+\frac{\sigma}{\pi}\right) \text {. }
$$

It generates the centering torque

$$
\tau=F c \tan \alpha=k c \tan \alpha\left((R-r)(1-\cos \alpha)+\frac{\sigma}{\pi}\right)
$$

of the system. The stiffness is

$$
\begin{aligned}
S= & \frac{d \tau}{d \varphi}=k c^{2}\left[-1+\frac{R-r+\frac{\sigma}{\pi}}{(R-r) \cos \alpha}+\right. \\
& \left.+\frac{\left(R-r+\frac{\sigma}{\pi}\right) c^{2} \varphi^{2}}{((R-r) \cos \alpha)^{3}}\right]
\end{aligned}
$$

and the potential energy stored in the system is

$$
\begin{aligned}
E= & \int_{0}^{\varphi} \tau d \varphi=-k\left[\frac{1}{2} c^{2} \varphi^{2}+\right. \\
& \left.+(R-r)\left(\left(1+\frac{\sigma}{\pi}\right) \cos \alpha+\frac{\sigma}{\pi}-R+r\right)\right] .
\end{aligned}
$$

The progressive shape of the cam disk forms an intrinsic protection of the system, which prevents the joint from running into the hardware limits. When they are reached, the spring mechanism is bypassed with a mechanical blocking. In this case the gear is the direct connection between the link and the motor inertia. A speed difference of motor and link then results in a torque peak, whose magnitude is depending on the gear flexibility. This torque peak of the inner system impact may cause serious damage to the system.

The system behavior with a deflection in positive direction is presented in Fig. 5. The system is built symmetrically 

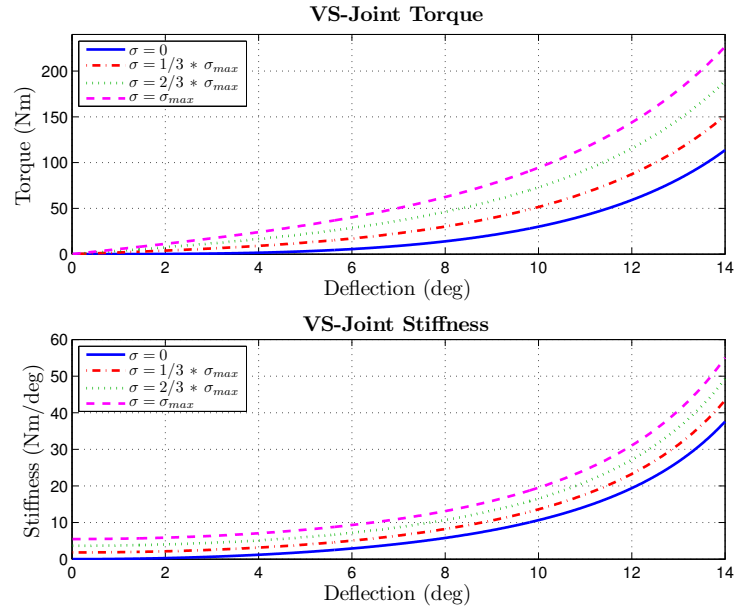

Fig. 5. System behavior concerning the joint torque and the joint stiffness.

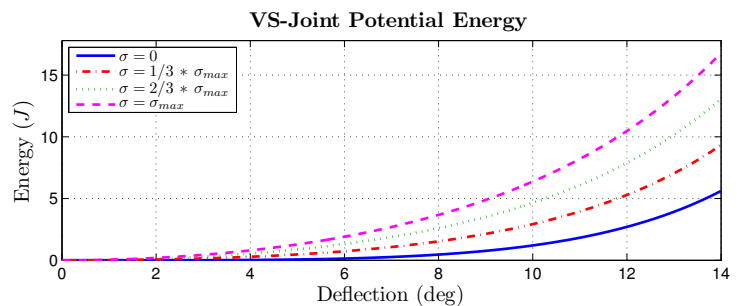

Ratio of Max./Min. Stiffness

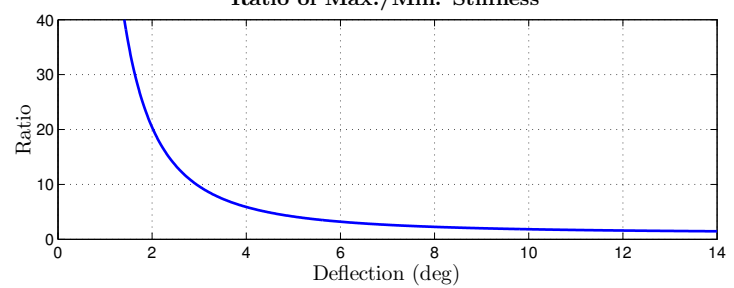

Fig. 6. Stored energy in the system and the ratio of maximum to minimum stiffness with respect to the deflection.

and has maximum deflection of $14^{\circ}$ in both directions. In contrast to antagonistic joints, the deflection range is not reduced by a higher stiffness preset. On a given deflection the stiffness of the joint and its derivative are under any condition higher with an increased stiffness adjuster position. The stiffness adjuster is able to change the preset continuously between minimum and maximum, and the power of the actuator enables the joint to change the preset bidirectionally under full load. The ratio of maximum to minimum stiffness is given in Fig. 6 and tends to infinity at zero deflection.

\section{TESTING SETUP}

The test bed (Fig. 7) consists of a motor / gear driving unit at the base side and a hollow shaft axle with a lever at the link side. The driving unit with a maximum output torque of $160 \mathrm{Nm}$ consists of a DLR ILM 70 motor attached through the hollow shaft link axle to a Harmonic Drive HFUS 20 (100/1). The CS of the Gear is mounted to the VS-Joint and the FS to the link axle. A DLR magnetoresistive (MR) sensor with a resolution of $30720 \mathrm{inc} / \mathrm{rev}$ is connected to the motor and a Heidenhain ERN120 with a resolution of $20000 \mathrm{inc} / \mathrm{rev}$ is attached to the link. A DLR torque sensor with a maximum

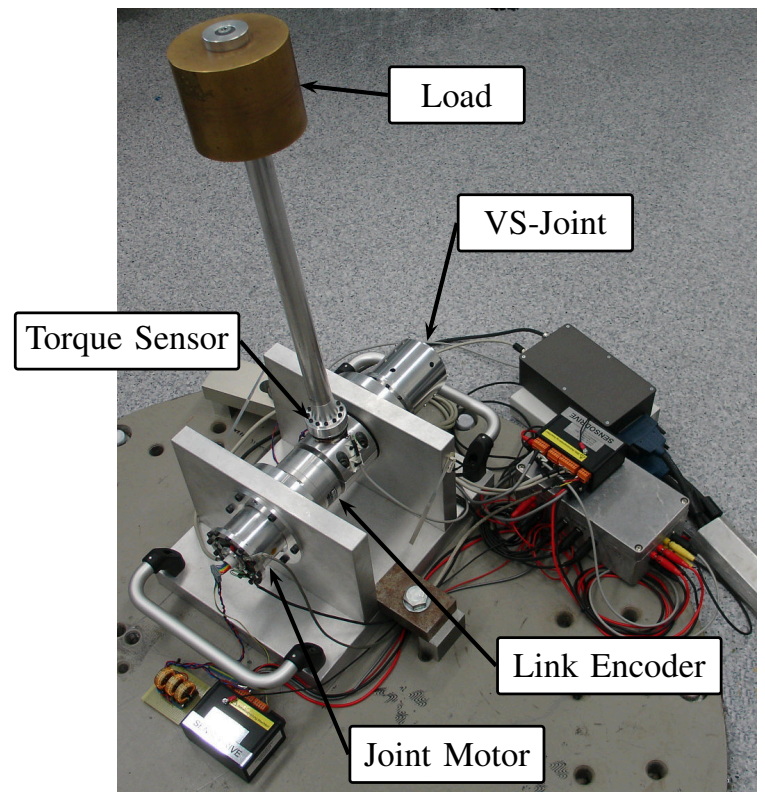

Fig. 7. Testing setup equipped with $7.0 \mathrm{~kg}$ load.

sensor range of $\pm 200 \mathrm{Nm}$ is mounted at the lever base. The sensor data acquisition is done by a National Instruments NI6602 and a NI6025E card. The motor controllers are two Sensodrive Unireg12 connected via CAN-Bus to a Softing CAN-AC2 card. The testbed is controlled by a computer with a QNX Software Systems real time operating system QNX ${ }^{\circledR}$ Neutrino ${ }^{\circledR}$. The control of the two motors is done position based with PD controllers.

The lever can be equipped with loads up to $7.0 \mathrm{~kg}$. Depending on the loads the link inertia varies between $0.124 \mathrm{~kg} \mathrm{~m}^{2}$ and $2.133 \mathrm{~kg} \mathrm{~m}^{2}$. In the further tests the upright position of the lever is defined as the zero position and the positive displacement is in the mathematical positive direction seen from the joint motor side.

\section{TESTS \& DEMONSTRATIONS}

\section{A. Evaluation}

The evaluation of the torque model, which is based on (5), was done with a fixed link at the end of the lever. In that setup the calculated torque is evaluated with the torque sensor. The theoretical torque does not include the strain of the joint structure. The spring base slider, the spindle connected to it, and the linear bearings do have notable flexibility, which can not be neglected. The strain was identified to be linear to the applied torque and results in a factor of 0.78 to the calculated torque. Fig. 8 shows the estimated torque including the correction factor plotted against the sensor data. The test trajectory of the joint motor is a position ramp, in which the joint is moving with a constant velocity between the joint deflection limits. This is done with minimum and maximum stiffness preset. The plot shows a very good linear correlation with a small hysteresis, which is the result of friction and sensor hysteresis.

A crucial factor to the system performance is the change of the stiffness preset. The time to change the stiffness is 


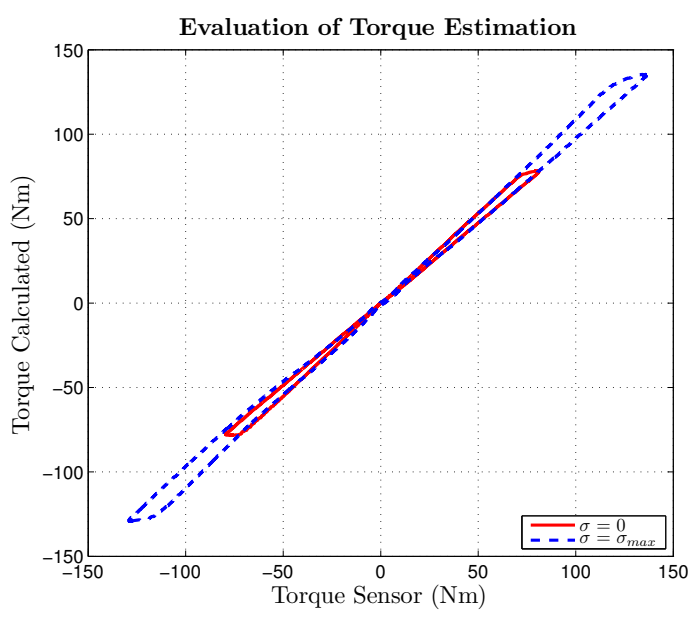

Fig. 8. Estimated Torque with respect to measured torque of the sensor.

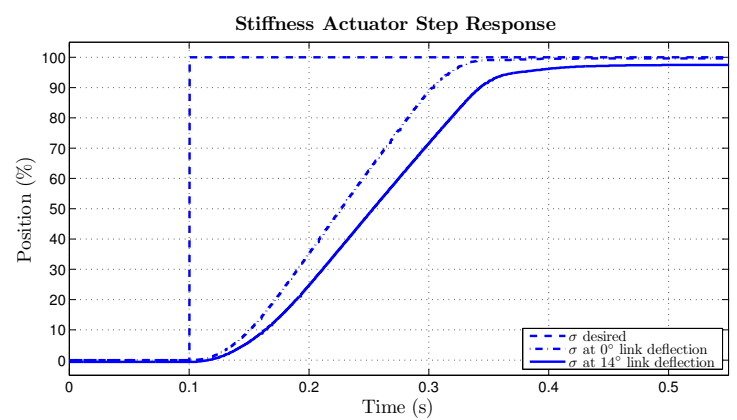

Fig. 9. Step response of the stiffness actuator from minimum to maximum with a fixed joint end at $0^{\circ}$ and $14^{\circ}$ joint deflection

decisive for the tasks and control of the system. In order to increase the stiffness preset the stiffness actuator has to compress the springs by moving the spring base slider. An external load also results in a compression of the springs so that the force applied to the spring base slider is increased. According to this the critical movement is an increase with external load applied. A step response with a fixed joint is given in Fig. 9. The steady state error in the test run with maximum joint deflection is a result of the PD controller, which is currently used for the stiffness adjuster motor.

\section{B. Throwing}

The application of throwing a ball is a good example to show the performance enhancement gained by the VS-joint in terms of maximum link velocity. For throwing a ball as far as possible it has to be accelerated to the maximum velocity and released at an angle of $45^{\circ}$. The link velocity of a stiff joint corresponds to the velocity of the driving motor. In a flexible joint the potential energy stored in the system can be used to accelerate the link relative to the driving motor. Additional energy can be inserted by the stiffness adjuster of the variable stiffness joint to gain the fastest possible motion.

A lacrosse stick head was mounted to the top of the link lever for the throwing tests, see Fig. 10 and the accompanying video. The ball is a $64 \mathrm{~g}$ rubber ball for school lacrosse. The distance $l$ between the link axis and the center of the

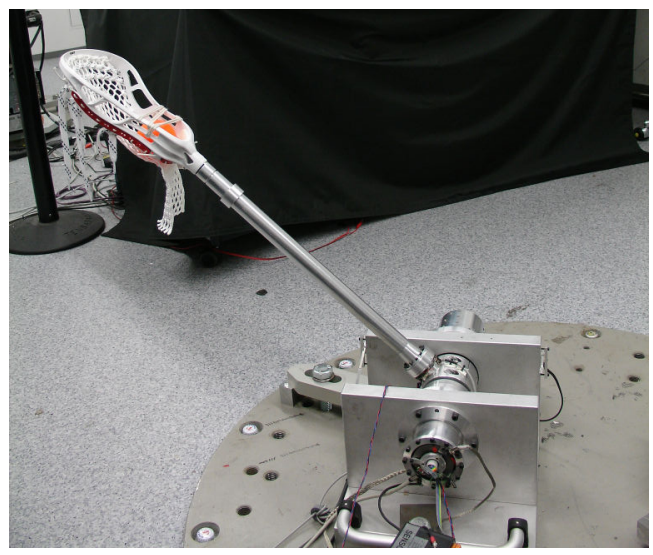

Fig. 10. Throwing setup with a lacrosse stick head mounted to the top of the lever.
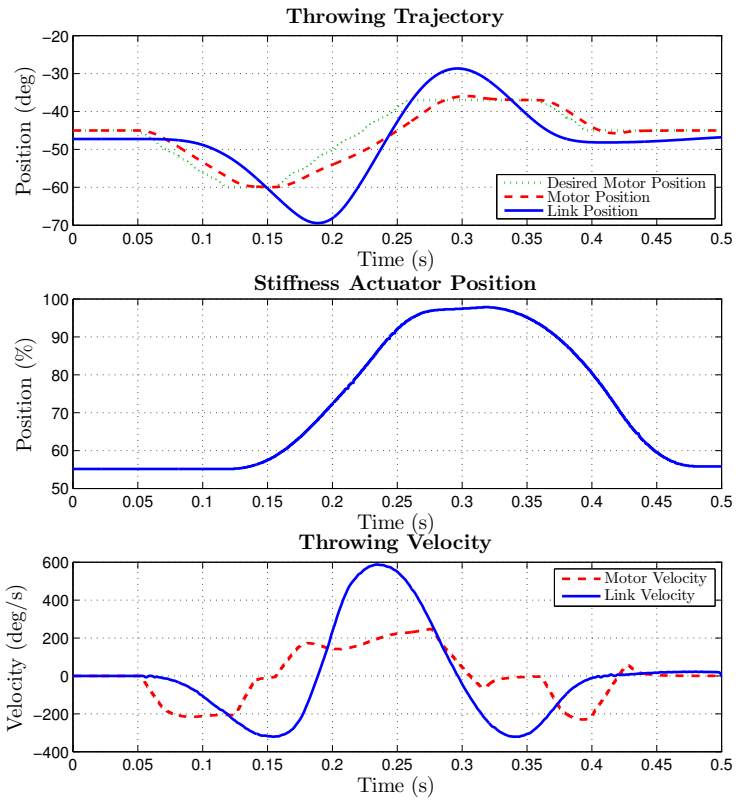

Fig. 11. A strike out trajectory of the joint motor in combination with an increase of the stiffness preset are used to gain maximum link velocity.

ball when the ball leaves the lever is approximately $0.78 \mathrm{~m}$.

A simple strike out trajectory is used for the demonstration in order to gain a high link velocity (Fig. 11). A joint motor position ramp accelerates the link in the negative direction to add kinetic energy to the link. When the motor stops and reverts, this energy is transformed into potential energy stored in the VS-Joint. The stiffness adjuster starts moving to the maximum pretension, which additionally increases the potential energy of the system. The effect can be seen in Fig. 6. The stored energy in the system at the same joint deflection is higher with an increased stiffness actuator position. Afterwards the joint motor accelerates in the positive direction and adds the kinetic energy to the stored energy in the VS-Joint. When the joint motor reaches its maximum speed the link is further accelerated by the potential energy.

As long as the link is accelerating, the ball can not be faster than the link, and the shape of the lacrosse stick head 
prevents the ball from leaving it in radial direction. This leads to the assumption, that the ball is leaving the lacrosse stick head at the maximum link velocity $\dot{q}_{\text {max }}$. In this case the velocity of the ball is

$$
v=\dot{q}_{\max } l .
$$

With a throwing angle $\beta$ relative to the ground and the gravity $g$, the range of throwing the ball is given by

$$
X=\frac{v^{2}}{g} \sin (2 \beta) \text {. }
$$

With the measured maximum link velocity (see Fig. 11) of $588 \%$ s, the throwing distance of the given example is

$$
X_{\mathrm{VS}-\text { Joint }}=\frac{\left(8.0 \mathrm{~m} \mathrm{~s}^{-1}\right)^{2}}{9.81 \mathrm{~m} \mathrm{~s}^{-2}} \sin \left(\frac{\pi}{2}\right)=6.52 \mathrm{~m} \text {. }
$$

The throwing distance of this demonstration could not be measured because the size of the available lab is not sufficient.

The theoretical throwing distance with a inelastic joint of the same setup with the same maximum joint motor velocity of $216 \%$ is

$$
X_{\text {fixed }}=\frac{\left(2.94 \mathrm{~m} \mathrm{~s}^{-1}\right)^{2}}{9.81 \mathrm{~m} \mathrm{~s}^{-2}} \sin \left(\frac{\pi}{2}\right)=0.88 \mathrm{~m},
$$

which was roughly confirmed in experiments, see the accompanying video.

A speed gain of $272 \%$ for the link velocity between rigid and compliant joint was achieved in the test.

Compared to a human the throwing range of the VS-Joint seems small, but one has to keep in mind that this was done by a single joint whereas a human uses several degrees of freedom. A series arrangement of joints in a robot arm promises to enhance the achievable distance.

\section{CONCLUSIONS \& FUTURE WORKS}

The main target of the work shown here is the development of a robot joint with a variable stiffness from scratch. Compared to state-of-the-art joints the new mechanical construction has a light-weight, compact, and powerful design. The passive mechanical compliance can be easily adapted to different applications by changing only the cam disk of the joint. The mechanical compliance acts as a storage for potential energy. This can be utilized as a mechanical buffer to reduce peak torques in the system and thus enhance the system safety, but it can also enhance the joint performance to gain a much higher link velocity than the maximum joint motor velocity. The joint has been evaluated on a testing setup and has proofed to have low friction and high stiffness adjusting dynamics. The velocity performance gain of the joint is presented in a throwing demonstration.

Future work includes an advanced control on the link position including active damping. The energy consumption of the system with its two differently sized motors has to be evaluated in various tasks to be compared to other systems including antagonistic principles. The ideal passive mechanical compliance has to be identified depending on the payload, dynamic requirements and the complete robot arm system setup.

\section{ACKNOWLEDGMENTS}

The authors acknowledge the support of Alin AlbuSchäffer, Markus Grebenstein and Werner Friedl. The authors give profuse thanks to Sami Hadaddin for his help on the throwing demonstration.

\section{REFERENCES}

[1] M. Grebenstein and P. van der Smagt, "Antagonism for a highly anthropomorphic hand-arm system," Advanced Robotics, 2008, in print.

[2] G. A. Pratt and M. M. Williamson, "Series elastic actuators," in Proc. of the IEEE/RSJ International Conference on Intelligent Robots and Systems. Pittsburg, PA, USA: IEEE/RSJ, July 1995, pp. 399-406.

[3] A. Bicchi and G. Tonietti, "Fast and soft-arm tactics - dealing with the safety-performance tradeoff in robot arms design and control," IEEE Robotics \& Automation Magazine, vol. June 2004, pp. 22-33, June 2004.

[4] K. Koganezawa, "Mechanical stiffness control for antagonistically driven joints," in Proc. of the IEEE/RSJ International Conference on Intelligent Robots and Systems. IEEE/RSJ, August 2005, pp. 25122519.

[5] S. A. Migliore, E. A. Brown, and S. P. DeWeerth, "Biologically inspired joint stiffness control," in Proc. 2005 IEEE International Conference on Robotics and Automation. Laboratory for Neuroengineering, Georgia Institute of Technology, Atlanta, Georgia 30332: IEEE, April 2005, pp. 4519-4524.

[6] C. English and D. Russell, "Implementation of variable joint stiffness through antagonistic actuation using rolamite springs," Mechanism and Machine Theory, vol. 34, no. 1, pp. 27-40, 1999.

[7] G. Tonietti, R. Schiavi, and A. Bicchi, "Design and control of a variable stiffness actuator for safe and fast physical human/robot interaction," in Proc. 2005 IEEE International Conference on Robotics and Automation. Interdepartmental Research Center E. Piaggio Faculty of Engineering, University of Pisa via Diotisalvi,2 56100, Pisa (Italy): IEEE, April 2005, pp. 582-533.

[8] M. Zinn, O. Khatib, B. Roth, and J. K. Salisbury, "Playing it safe - a new actuation concept for human-friendly robot design," IEEE Robotics \& Automation Magazine, vol. June 2004, pp. 12-21, June 2004.

[9] T. Morita and S. Sugano, "Development and evaluation of seven-d.o.f. mia arm," in Proc. 1997 IEEE International Conference on Robotics and Automation, September 1997, pp. 462-467.

[10] M. Okada, Y. Nakamura, and S. Ban, "Design of programmable passive compliance shoulder mechanism," in Proc. 2001 IEEE International Conference on Robotics \& Automation. Dept. of MechanoInformatics, University of Tokyo 7-3-1 Hongo Bunkyo-ku Tokyo, 113-8656 Japan; CREST Program, Japan Science and Technology Corporation: IEEE, May 2001, pp. 348-353.

[11] S. Haddadin, A. Albu-Schäffer, and G. Hirzinger, "Safety evaluation of physical human-robot interaction via crash-testing," in Robotics: Science and Systems Conference (RSS2007), 2007.

[12] B. Vanderborght, B. Verrelst, R. V. Ham, M. V. Damme, D. Lefeber, B. M. Y. Duran, and P. Beyl, "Exploiting natural dynamics to reduce energy consumption by controlling the compliance of soft actuators," The International Journal of Robotics Research, vol. 25, no. 4, pp. 343-358, 2006.

[13] J. W. Hurst, J. E. Chestnutt, and A. A. Rizzi, "An actuator with physically variable stiffness for highly dynamic legged locomotion," in Proc. of IEEE International Conference on Robotics and Automation. New Orleans, LA, USA: IEEE, April 2004, pp. 4662-4667.

[14] D. P. Ferris, M. Louie, and C. T. Farley, "Running in the real world adjusting leg stiffness for different surfaces," Proceedings of the Royal Society B: Biological Sciences, vol. 265, no. 1400, pp. 989-994, June 1998.

[15] C. Ott, O. Eiberger, W. Friedl, B. Bäuml, U. Hillenbrand, C. Borst, A. Albu-Schäffer, B. Brunner, H. Hirschmuller, S. Kielhofer, R. Konietschke, M. Suppa, T. Wimbock, F. Zacharias, and G. Hirzinger, "A humanoid two-arm system for dexterous manipulation," in 6th IEEE-RAS International Conference on Humanoid Robots, Genova, December 2006, pp. 276-283. 